\title{
PROCESS AND SYSTEMS Reducing readmission rates through a discharge follow-up service
}

\author{
Authors: Duncan Vernon, ${ }^{\mathrm{A}}$ James E Brown, ${ }^{\mathrm{B}}$ Eliza Griffiths, ${ }^{\mathrm{C}}$ Alan $\mathrm{M}$ Nevill ${ }^{\mathrm{D}}$ and Martha Pinkney ${ }^{\mathrm{E}}$
}

Approximately $15 \%$ of elderly patients are readmitted within 28 days of discharge. This costs the NHS and patients. Previous studies show telephone contact with patients post-discharge can reduce readmission rates. This service evaluation used a cohort design and compared 30-day emergency readmission rate in patients identified to receive a community nurse follow-up with patients where no attempt was made. 756 patients across seven hospital wards were identified; 303 were identified for the intervention and 453 in a comparison group. Hospital admission and readmission data was extracted over 6 months. Where an attempt to contact a patient was made post-discharge, the readmission rate was $9.24 \%$ compared to $15.67 \%$ where no attempt to contact was made $(p=0.011)$. After adjustment for confounding using logistic regression, there was evidence of reduced readmissions in the 'attempt to contact' group odds ratio $=1.93(95 \%$ confidence interval $=1.06-3.52, p=0.033$ ). Of the patients who community nurses attempted to contact, 288 were contacted, and 202 received a home visit with general practitioner referral and medications advice being the most common interventions initiated. This service evaluation shows that a simple intervention where community nurses attempt to contact and visit geriatric patients after discharge causes a significant reduction in 30-day hospital readmissions.

KEYWORDS: Discharge, geriatrics, readmission, telephone contract

\section{Introduction \\ Patient recruitment}

It is recognised that the number of emergency readmissions is rising. NHS data shows that in 2016/17 there were 529,318 emergency readmissions reported by 84 hospital trusts and that over the past 4 years this has risen by almost $25 \%$. The elderly are more likely to be readmitted and $15 \%$ of over 65 -year-olds

Authors: ${ }^{A}$ speciality registrar in public health, Solihull Metropolitan Borough Council, Solihull, UK; B senior lecturer in biosciences, Aston University, Birmingham, UK; ${ }^{C}$ consultant geriatrician, South Warwickshire Foundation Trust, Warwick, UK; D professor of biostatistics, University of Wolverhampton, Walsall, UK; ${ }^{\mathrm{E}}$ consultant geriatrician, Solihull Hospital, Solihull, UK in England are readmitted within 28 days. ${ }^{2}$ There are many risk factors for readmission but longer hospital stays or a history of admissions are more common among readmitted patients. Functional disability and morbidity are also notable risk factors. ${ }^{3}$

There is no consensus about what proportion of readmissions are avoidable. A systematic review of 34 studies reported a median proportion of avoidable readmission of $27.1 \%$, however there was a large amount of heterogeneity between studies and the range was between 5 and $79 \% .{ }^{4}$ A typology of emergency readmissions in England identified that 30\% were due to probable or possible suboptimal care during the initial hospital spell. Conversely, $19 \%$ were most likely to be due to an accident or coincidence unrelated to the initial admission. ${ }^{5}$

A systematic review of randomised controlled trials identified that interventions to prevent readmissions were more effective when they had multiple components, such as including more than one person involved in the delivery of the intervention or increasing patients' ability to care from themselves. ${ }^{6}$

Some interventions have been evaluated separately and identified as effective, with the care received after discharge influencing readmission rates. Medical patients in contact with a social worker, referred to a community health professional or receiving patient education were less likely to be readmitted. ${ }^{7}$

There is promising evidence that a telephone call following discharge reduces readmissions by up to $50 \% .{ }^{8-10}$ However a Cochrane review in 2006 was unable to draw conclusions due to heterogeneity of research and low methodological quality. ${ }^{11}$

The importance of follow-up after discharge is also recognised in National Institute for Health and Care Excellence guidance, which recommends that patients with social care needs who are at risk of readmission receive a follow-up phone call or visit from either a community nurse or general practitioner (GP) 24-72 hours after discharge. $^{12}$

This may also be an effective strategy when offered to all elderly patients. Heart of England Foundation NHS Trust (HEFT), of which Solihull hospital is part, designed a novel service where patients were offered a visit from a band 6 community nurse, after an initial phone call within 48 hours after discharge. The service was designed to assess patients' needs quickly after discharge and prevent readmissions.

The community nurses identified patients based on discharge lists, which would prevent any delays in follow-up arising from poor quality or delayed information to the GP. ${ }^{13}$

The aim of this service evaluation was to understand whether this contact from a community nurse following discharge, and resulting 
decisions about what support the patient needed, could be effective in reducing readmissions among all elderly medical patients.

\section{Methods}

This study is a pragmatic service evaluation, making use of a comparison group to create a cohort study. Comparison groups are a powerful method of identifying whether an intervention is effective. ${ }^{14,15}$ The study followed the STrengthening the Reporting of OBservational studies in Epidemiology (STROBE) guidelines for presenting a cohort study.

\section{Patient recruitment}

Patients aged over 65 and registered at a GP that was a member of the Solihull Clinical Commissioning Group (CCG) were selected to receive the intervention. Seven hospital wards took part in the trial across two sites. These wards were a mixture of elderly care and medical specialty wards. Patients in the intervention group were identified by checking the discharge lists for the participating wards daily. Patients discharged to hospice care, nursing homes or residential homes were excluded from the trial at recruitment, as there was a separate care home nursing team providing a service to these patients.

A comparison group was identified from the same source and included all patients aged over 65 discharged from the same wards as the intervention group but registered at a GP member of a Birmingham CCG. Patients discharged to nursing or residential care or hospice care were also excluded from this group. No matching criteria were used on recruitment.

With the exception of patients who did not meet the inclusion criteria, all of the patients eligible to take part were recruited to the cohorts. To restrict bias, the intervention and comparison group was restricted to patients receiving care on the same wards, and all of the patients were in hospital due to unplanned admissions. Limiting the age of interest of over 65-year-olds also helps to reduce bias from potentially different age profiles.

\section{Intervention description}

Two band 6 nurses attempted to contact the patients by telephone within 48 hours of discharge. Multiple attempts were made within 48 hours of discharge. When contact was made, patients were given the opportunity to discuss issues arising after discharge and were offered a home visit by one of the two nurses. The visits were an opportunity to explore issues identified in the telephone call and to further assess patient needs. The community nurses recorded which patients they visited, and what actions or referrals they made following the visit.

The community nurses had access to a consultant community geriatrician who provided clinical support and could also see patients on home visits or in rapid access clinic slots for comprehensive geriatric assessment. A community pharmacist was available as an additional resource for queries regarding medication side effects and interactions. The nurses could refer on to other NHS services based on their assessment of the patient's needs.

\section{Data collection and statistical analysis}

The same process was used to collect outcome data for patients in both the intervention and comparison groups. Data on hospital admissions and readmissions for patients who received the intervention, and the comparison group, were extracted from the HEFT computer system (icare) between the dates of 01 January 2016 and 30 June 2016, a month after the conclusion of the 6 month trial. A spreadsheet was designed to extract data from hospital records on admission date, discharge date, date of emergency readmission, length of readmission, age (within 5-year band), gender, hospital, ward and home postcode. The community nurses kept records on what intervention patients received during the visit. The data in the hospital records was complete for all of the variables of interest.

Patients who died during the 30-day follow-up period were identified using the hospital records system. There was no way of tracking readmissions to other hospitals or if a patient had moved out of the area within 30 days.

The primary outcome measure for this study was an emergency readmission within 30 days of the original discharge. Patients with multiple readmissions were assessed on the first readmission only.

Statistical analysis was performed using SPSS 22 software (SPSS Inc, USA). Readmission rate was explored using backwards stepwise binary logistic regression.

The extracted data set was used to create covariables to include in the statistical analysis, including the age of the patients in 5 -year age bands, gender, the Index of Multiple Deprivation (IMD) score and hospital site where original discharge occurred. IMD scores were calculated from home postcode using the UK Ministry of Housing, Communities \& Local Government online tool (http:// imd-by-postcode.opendatacommunities.org).

The five explanatory variables included in the model were gender, age in years, IMD score as a categorical variable based on deprivation decile, attempt to contact and hospital site. Crosstabulations were used with the associated chi-squared tests.

\section{Ethics and funding}

The pilot was offered to all patients who were registered with a GP practice which was part of the same CCG. There was no randomisation and all patients received as a minimum the standard care commissioned by their local CCG. Data was extracted from hospital records. The NHS Health Research Authority decision-making tool classified this as a service evaluation which does not need approval from an ethics committee (http://hra-decisiontools.org.uk/ethics/). Funding for the two nurses was provided by the NHS trust, there was no additional funding to conduct the evaluation.

\section{Results}

\section{Characteristics of the patients included}

Seven-hundred and seventy-five patients were identified during the 6 months that the service ran. Nineteen of these patients were excluded before analysis due to death during the 30-day follow-up period (seven patients), inappropriate age (10 patients) or no age recorded (two patients). Of the remaining patients, community nurses attempted to contact 303 to offer a home visit, and 453 made up the comparison group. When comparing the baseline characteristics of the intervention and comparison group, there were statistically significant differences between groups for IMD $(p<0.001)$ with more deprived subjects in the intervention group, hospital $(p<0.001)$ with more subjects in the contact group being discharged from the larger of the two sites, age $(p=0.018)$ with the 
contact group being younger. There was no significant difference in gender between groups.

At the time of discharge, the average age of patients in the intervention group was 78.65 years old ( \pm 7.74 years), with 115 males and 188 females. The median IMD decile was 6 . In the comparison group, the average age was 80.08 years old ( \pm 7.83 years), with 188 males and 267 females. The median IMD decile was 2 .

Across both groups there were a total of 99 30-day emergency readmissions. Seventy-one readmissions occurred in the comparison group and 28 in the intervention group.

\section{Interventions and referrals initiated by the visit}

Of the 303 patients in the intervention group, there was successful telephone contact with 288 , and 202 received a home visit. Table 1 shows the interventions provided and further referrals. Each patient may have received more than one intervention.

\section{Interaction between attempt to contact and readmission}

Follow-up using hospital records was conducted over 30 days after the intervention to ensure complete follow-up of the primary outcome. The relationship between readmissions and whether the patient was in the intervention or comparison group can be seen in Table 2. Patients in the intervention group had a significantly lower readmission rate. The frequency of patients readmitted within 30 days was significantly less in the group where an attempt was

Table 1. Interventions and referrals initiated by receiving the new service

\begin{tabular}{ll} 
Intervention & $\begin{array}{l}\text { Number of } \\
\text { patients }\end{array}$ \\
Advice & \\
Medication support/advice & 75 \\
Incontinence assessment & 1 \\
Continence advice & 3 \\
Referral & \\
General practitioner & 66 \\
Pharmacist & 15 \\
Rapid response community nursing service & 8 \\
Mental health nurse & 18 \\
Geriatrician & 7 \\
Equipment & 28 \\
Social services & 3 \\
District nurse & 1 \\
Foot health & 1 \\
Heart failure nurse & 1 \\
Physiotherapy & 3 \\
Occupational therapy & 3 \\
Falls team & 3 \\
Respiratory nurse & 1 \\
No advice/referral & 3 \\
\hline
\end{tabular}

\begin{tabular}{|c|c|c|c|c|}
\hline \multirow{4}{*}{ Readmitted, $\mathrm{n}$} & & \multicolumn{2}{|c|}{ Contact, n } & \multirow[t]{2}{*}{ Total, $n$} \\
\hline & & No & Yes & \\
\hline & No & 382 & 275 & 657 \\
\hline & Yes & 71 & 28 & 99 \\
\hline Total, $n$ & & 453 & 303 & 756 \\
\hline Per cent of total, \% & & 15.67 & 9.24 & - \\
\hline
\end{tabular}

made to contact $(9.24 \%)$ than in the group where no attempt to contact was made (15.67\%; Fisher's exact test (1df), $p=0.011$ ). For the readmitted-by-hospital site interaction the frequency of readmission from Solihull and Heartlands were not significantly different (15.3\% vs 12.3\%; Fisher's exact test (1df), $p=0.319$ ).

Patient age was categorised into 5 -year bands and IMD was grouped into deciles for the purposes of the analysis.

\section{Independent predictors of readmissions}

Further analysis was performed to identify which independent variables could predict 30-day readmission rate. Binary linear regression revealed 'attempt to contact' as a significant predictor of readmission (odds ratio $(\mathrm{OR})=1.825,95 \%$ confidence interval $(\mathrm{CI})=$ 1.148-2.913, $p=0.011$ ). When all confounding factors were included in our analysis, 'attempt to contact' is still a significant predictor of hospital readmission $(\mathrm{OR}=1.927, \mathrm{CI}=1.056-3.517, \mathrm{p}=0.033)$. These data suggest that where no attempt was made to contact patients, they had a 1.927-fold higher relative chance of emergency readmission compared to those where an attempt to contact was made.

\section{Discussion}

With an ageing population and pressure on health services, it is important to identify how to avoid unnecessary hospital readmissions. This service evaluation identified that individuals in receipt of a follow-up from a community nurse were almost twice as likely to avoid a 30-day readmission compared to patients where no attempt was made. This shows that a simple and novel intervention can dramatically reduce readmission rates. The decrease was statistically significant and could not be explained by differences between the two groups of patients.

Within the intervention group, the attempt to contact was successful in $95 \%$ of cases, and $67 \%$ of patients in the group resulted in a home visit. While a GP referral and medication advice were the most common intervention received, the number of different types of referral or interventions demonstrates the advantage of an approach that assesses patient needs in their home environment.

The scale of the reduction was similar to another study that found telephone calls following discharge reduced readmissions by $50 \%{ }^{8}$ There is also some consistency with research showing that providing a home visit to high-risk patients with complex needs following discharge was effective at reducing readmissions. ${ }^{16}$ The complexity of identifying which specific interventions offered by the community nurses were effective was beyond the size and power of this study.

The study was essentially a cohort design, which may have introduced unknown confounding factors for which we were 
unable to account through statistical methods in the analysis. Our choice of recruiting the comparison group from the same wards may have had an impact on this. ${ }^{17}$ The nature of CCG boundaries meant that practically the two groups were drawn from separate but overlapping geographic areas. This was potentially a reason for the significant differences in baseline characteristics between the two groups, although these factors did not materially change the conclusion after adjustment.

As our comparison group was drawn from the same wards as the patients receiving the intervention, this will have also reduced the likelihood of some confounding factors by limiting participation in the study to elderly patients in need and receipt of comparable levels of hospital care.

Although we were able to adjust for the confounding factors collected in the analysis, other unknown or residual confounding factors may reduce the effect size further. This includes history of admissions or other measures of patient health that may have only been partially accounted through inclusion of patient age and geographic factors such as IMD in our model.

The use of another CCG's patients also introduces a potential confounding variable if other community services are different. However, the readmission rates in over 65 -year-olds for both sites were broadly similar to national rates. A further weakness was the reliance in the data from two hospitals in one provider trust. However, most readmissions are likely to be to the same hospital as discharge.

There is a potential misclassification bias in that patients who could have received the intervention but were not contacted are included in the exposure group. This gives an effect size that may be a closer estimate of introducing the intervention in the real world where some patients are unable to engage with the service. The short nature of the study and exclusion of patients who died reduces the risk of loss to follow-up in the cohort.

Despite these weaknesses, the study shows promising early findings that reductions in readmission rates could be achieved by offering community nurse follow-up after discharge. The setting is similar to many NHS trusts and makes use of community nurses in a targeted manner.

The findings also suggest that further studies will need to look at the wider impacts and costs of delivering this service. These impacts include sustainability of interventions outside of the secondary care setting, for instance increased community and primary care service activity. Other outcome measures related to the patients' health and wellbeing will also be important in future studies.

The use of more robust study designs such as a randomised controlled trial would allow for the collection of higher quality data around the nature of the readmission and what happens after referral. This would also help to identify those readmissions that may be most preventable.

\section{Conclusion}

In conclusion, we have shown that a simple intervention whereby community nurses attempt to contact geriatric medical patients after discharge is a promising way to reduce 30-day hospital readmission rate.

\section{Funding}

Funding for the intervention was provided on a pilot basis by Heart of England Foundation Trust, there was no additional funding to conduct the evaluation.

\section{References}

1 Healthwatch. NHS needs to do more to understand why people are returning to hospital after being discharged. London: Healthwatch, 2017. www.healthwatch.co.uk/news/nhs-needs-do-more-understand-why-people-are-returning-hospital-after-being-discharged.

2 Oliver D. David Oliver: Who is to blame for older people's readmission? BMJ 2015;351:h4244.

3 Garcia-Perez L, Linertova R, Lorenzo-Riera A et al. Risk factors for hospital readmissions in elderly patients: a systematic review. QJM 2011;104:639-51.

4 van Walraven C, Bennett C, Jennings A, Austin PC, Forster AJ. Proportion of hospital readmissions deemed avoidable: a systematic review. CMAJ 2011;183:E391-402.

5 Blunt I, Bardsley M, Grove A, Clarke A. Classifying emergency 30-day readmissions in England using routine hospital data 2004-2010: what is the scope for reduction? Emerg Med J 2015;32:44-50.

6 Leppin AL, Gionfriddo MR, Kessler M et al. Preventing 30-day hospital readmissions: a systematic review and meta-analysis of randomized trials. JAMA Intern Med 2014;174:1095-107.

7 Chan MF, Wong FK. The risk factors for hospital re-admission in medical patients in Singapore. Health Soc Care Community 2014:22:488-96.

8 Dudas V, Bookwalter T, Kerr KM, Pantilat SZ. The impact of follow-up telephone calls to patients after hospitalization. Am J Med 2001;111:26S-30S.

9 Stewart S, Pearson S, Luke CG, Horowitz JD. Effects of home-based intervention on unplanned readmissions and out-of-hospital deaths. J Am Geriatr Soc 1998;46:174-80.

10 Burns ME, Galbraith AA, Ross-Degnan D, Balaban RB. Feasibility and evaluation of a pilot community health worker intervention to reduce hospital readmissions. Int ] Qual Health Care 2014;26:358-65.

11 Mistiaen P, Poot E. Telephone follow-up, initiated by a hospitalbased health professional, for postdischarge problems in patients discharged from hospital to home. Cochrane Database Syst Rev 2006:CD004510.

12 National Institute for Health and Care Excellence. Transition between inpatient hospital settings and community or care home settings for adults with social care needs. NICE guideline [NG27]. London: NICE, 2015. www.nice.org.uk/guidance/ng27/chapter/ Recommendations\#discharge-from-hospital.

13 Kripalani S, LeFevre F, Phillips CO et al. Deficits in communication and information transfer between hospital-based and primary care physicians: implications for patient safety and continuity of care. JAMA 2007;297:831-41.

14 Steventon A, Bardsley M, Billings ], Georghiou T, Lewis GH. The role of matched controls in building an evidence base for hospitalavoidance schemes: a retrospective evaluation. Health Serv Res 2012;47:1679-98.

15 Roland M, Dusheiko M, Gravelle H, Parker S. Follow-up of people aged 65 and over with a history of emergency admissions: analysis of routine admission data. BMJ 2005;330:289-92.

16 Scott IA. Preventing the rebound: improving care transition in hospital discharge processes. Aust Health Rev 2010;34:445-51.

17 Steventon A, Grieve R, Sekhon JS. A comparison of alternative strategies for choosing control populations in observational studies. Health Serv Outcomes Res Methodol 2015;15:157-81.

Address for correspondence: Dr Martha Pinkney, Heart of England NHS Foundation Trust, Solihull Hospital, Lode Lane, Solihull B91 2JL, UK.

Email: martha.pinkney@heartofengland.nhs.uk 